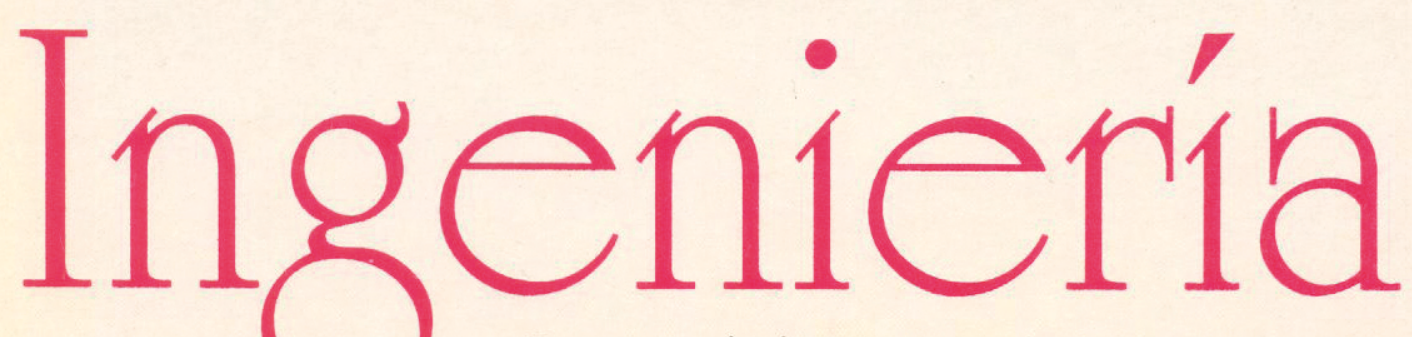

Revista de la Universidad de Costa Rica ENERO/JUNIO 1994 VOLUMEN 4 N 1

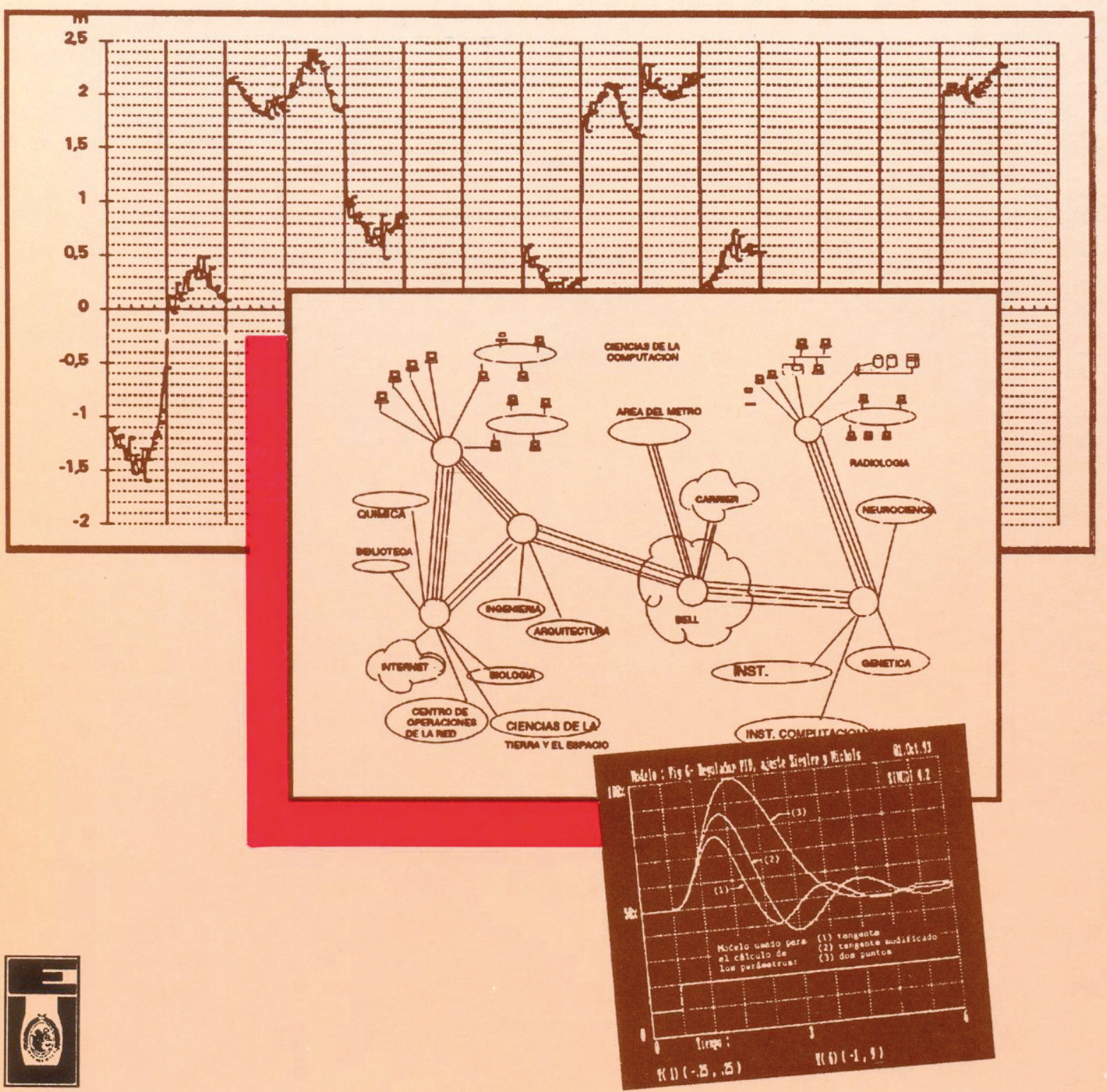




\title{
MODELANDO FLUJO UNIDIMENSIONAL NO ESTABLE EN CANALES DE ANCHO Y PROFUNDIDAD VARIABLE
}

\author{
Ing. Luis M. Murillo B.,Ph.D*
}

\section{RESUMEN}

\begin{abstract}
Se introduce un modelo hidrodinámico no estable que reproduce flujos unidimensionales en canales cuyo ancho, profundidad y longitud puede variar. El modelo es de uso en la predicción de inundaciones en ríos sujetos a cambios temporales drásticos como los que ocurren en las costas debido a las mareas y otros fenómenos hidráulicos transientes. En especial el modelo puede servir para predecir el efecto de puentes y otras obstrucciones al libre flujo y reflujo de las aguas. El modelo se aplica al flujo unidimensional que ocurre en la parte superior del Golfo de Nicoya y se reproducen los fenómenos más sobresalientes como la amplificación inercial y las corrientes.
\end{abstract}

\section{SUMMARY}

An unsteady, one-dimensional hydrodynamic model is introduced capable of reproducing variable flow conditions in channels ofvariable depth, width and length. The model can be used to propagate floods and other non steady state flows cornmon in coastal regions and account for changes to the basic geometry ofthe waterway required for cornmon civil works. The model reproduces the main known characteristics of flow in the upper Nicoya Gulf, Costa Rica. with its inertial amplification and variable current regime.

\section{INTRODUCCIÓN}

El cálculo del flujo no estable en ríos y canales abiertos producido por ondas largas y otros fenómenos hidráulicos no permanentes como propagación de inundaciones, es de gran importancia y utilidad para la ingeniería hidráulica yel desarrollo y utilización de los recursos costeros. Este flujo variable se puede reproducir muy exactamente mediante modelos hidrodinámicos unidimensionales de gran auge en la ingeniería moderna.

En este artículo se introduce un modelo capaz de reproducir el flujo no estable en canales de sección transversal variable. Dado un río cualquiera también es posible modelar los afectos de una obstrucción parcial creada por la construcción de un puente o toma de agua para irrigación. Aspectos estos de gran importancia para el manejo de los agotables recursos hídricos con que cuenta el país. Investigaciones anteriores sobre la hidrodinámica del Golfo de Nicoya, en la Costa Pacífica de Costa Rica (Murillo, 1982, Murillo, 1993) han estudiado el flujo no estable generado por las mareas. Los resultados indican que el flujo en la Región Supe- rior del Golfo de Nicoya posee características unidimensionales, con la onda propagándose y amplificándose inercial mente a lo largo del eje del Golfo. Sin embargo, se desconoce el modo de propagación en el límite superior del Golfo, en la desembocadura del río Tempisque y a lo largo de su cauce.

Se recuerdan con ansiedad una serie de inundaciones que ocurrieron en el río Tempisque hace pocos años. Aun hoy día se observan abundantes obras de protección construidas para protegerse de las temidas inundaciones.

Actualmente, se propone la construcción de un puente que atravesará el río Tempisque, unos kilómetros al norte de su desembocadura. La zonas aledañas son manglares y zonas de conservación natural que podrían verse fuertemente impactadas por un cambio de nivel de las aguas o un cambio de su velocidad y regímenes de sedimentación. Es necesario estudiar los posibles efectos hidráulicos que la construcción del puente puede tener.

Con este propósito y otros similares, es que se ha desarrollado el modelo hidrodinámico discutido aquí. 
La dificultad presente más seria radica en la ausencia de mediciones hidráulicas elementales, que sirvan de condiciones de frontera para el modelo tales como las como profundidades de varias secciones del río, sus áreas transversales, gastos y corrientes limítrofes en la zona del río Tempisque. Sin embargo se ofrece esta capacidad de modelaje para que el ingeniero hidráulico nacional, dedicado a resolver este problema del río Tempisque $\mathrm{u}$ otros similares que abundan en el país, tenga un instrumento de predicción y disefio base, al que se le puedan introducir los datos de campo conforme estos se vayan midiendo.

\section{ECUACIONES DIFERENCIALES DEL MODELO}

La ecuación de momento que gobierna el flujo inestable en ríos y canales se puede escribir como (Sabersky y Acosta, 1964)

$$
\frac{a Q}{\operatorname{atax}} \sim(\sim)_{A} \quad \frac{i 9 \mathrm{Am}-\mathrm{gA}(\mathrm{So}-\mathrm{St})}{\mathrm{ax}}=0
$$

con

$$
s_{f}=\frac{v^{2}}{c^{2} R_{L}}
$$

La ecuación de conservación de volumen es

$$
\frac{\mathrm{a} 0}{\mathrm{ax}}+\mathrm{B} \frac{\mathrm{a}}{\mathrm{at}}=\mathrm{O}
$$

El modelo resuelve las Ecuaciones (1) y (3) usando una mezcla de procedimientos numéricos bien conocidos en el ambiente ingenieril internacional: procedimientos de diferencias finitas hiperbólicos para las variaciones temporales, aceleraciones espaciales y la presión; procedimientos parabólicos para las aceleraciones temporales y el término de fricción. Los esquemas de solución numérica son implícitos Y la estabilidad de la solución se consigue adheriéndose a los límites establecidos por las líneas características de las ecuaciones anteriores (Abbott,1979); esto redunda para el río Tempisque en undiferencial temporal minimo de 60 minutos para segmentos de 1 kilómetro de largo.
El método de solución de diferencias finitas usa una malla con nodos temporales y espaciales no centrados, de tal forma, que los cómputos de nivel yvelocidad se realizan en distintos instantes. Las velocidades se calculan en cada sección transversal, pero las presiones en lugares intermedios. En las ecuaciones se substituyen los valores para el ancho de cada sección que puede ser variable. Los términos convectivos no lineales se evalúan directamante, por ejemplo:

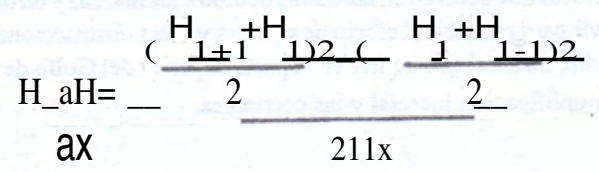

Los problemas relacionados con la dispersi6n numérica, típicos de ecuaciones hiperbólicas con varios armónicos en propagación, se tratan aquí usando esquemas de propagación de Frornm (Richtmyer R.D., Morton K.W, 1967) y procedimientos empíricos de ajuste de red.

Las condiciones de frontera usadas pueden ser de varios típos. Ya sea reflexión o absorción peñectas cuando la frontera sea cerrada. Cuando esta sea abierta, se usa una condición de propagación refractaria característica de ondas largas en ríos poco profundos o sea

$$
Q=H_{0} b \sqrt{(g d)}
$$

en donde Hoes el valor inmediato en la malla de cálculo.

El programa se escribe en Microsoft Fortran y se corre en computadores personales con coprocesador matemático. Durante la corrida el operador tiene la opción para visualizar resultados intermedios produciendo archivos ASCII que pueden ser leídos y graficados por medio de Hojas de Cálculo como 123, Qpro oExcel posteriormente.

\section{LA PROPAGACIÓN DE LA MAREA EN UN ESTUARIO UNIDIMENSIONAL}

Como ejemplo de aplicación del modelo (FLOOD .EXE), este se corrió para un estuario grande, climensionalmente igual al Golfo de Nicoya Superior, pero con profundidades de pendiente 
constante. La Función de Perturbación a la entrada (i.e en Puntarenas) está dada por una marea típica de la forma (Shureman. 1958).

$$
H=H_{0}+\sum_{1}^{7}\left(f_{1} A_{1} \cos \left(\omega t+\left(v_{0}+u\right)_{g}-\theta_{1}\right)\right.
$$

Los procedimientos para el cálculo de los argumentos de esta función se discuten en Murillo (1993). Se asume una pendiente de fondo homogénea e igual a 0.03. Los anchos se obtuvieron de un mapa a escala 1:100,000. El programa lee el archivo de iniciación (FLOOD.DAT) en donde se encuentran los datos de entrada tal y como se muestran en el Cuadro No 1.El inicio de los cálculos es el 3/4/ 1993 a las 6 amo Los resultados se imprimen cada 123 minutos. El coeficiente de Chézy usado fue de $30 \mathrm{mAl} / 2 / \mathrm{SEl}$ número de segmentos en que se dividió el Golfo fue de 30.

$$
\begin{gathered}
\text { Cuadro No. } 1 \\
\mathrm{t}=10, \sim=3600 \text { s. } \mathrm{x}=1600, \mathrm{H}_{\mathrm{O}}=\mathrm{O}, \mathrm{C}=30,1_{-}=30, Q \sim=6, \mathrm{M} . .=123, \text { Maxn=20500 } \\
\text { Afio: 1993. Mes: 4. Día: 3., Hora: 6. Minutos: } \mathrm{O} \\
\text { Anchos b(i) en } \mathrm{Km})
\end{gathered}
$$$$
4, .4, .6, .6, .6, .6, .7 r \quad 7, .7, .7, .7, .8, .9,1,1,1,1, .7, .5, .5, .5, .4, .5, .5, .5, .5, .4, .2, .2, .1
$$$$
\text { Pendientes del Fondo (Se\%) }
$$

Para esta corrida se usó una condición de frontera completamente reflectora en el extremo superior del GNS. Las velocidades enla entrada se calcularon según

$$
V=\operatorname{Hov}^{\prime}\left(\left\{\frac{g}{\mathrm{~d}}\right)\right.
$$

La Figura No. 1 muestra la superficie del GNS idealizada cada 123 minutos a partir del período 13, luego del 3/4/1993 a las 6:00 a.m. Se nota como la oscilación es casi simultánea en todo el Golfo pero con una amplitud variable. Laparte interior se amplifica significativamente con respecto a la función de perturbación a la entrada del estuario. La amplitud de la oscilación inercialmente amplificada es de $3.2 \mathrm{~m}$. a la entrada (primer segmento) y de unos $4.3 \mathrm{~m}$. en elúltimo segmento (Puerto Moreno en el interior del Golfo), 10 cual da un coeficiente de amplificación neto de $34 \%$.

La Figura No. 2 muestra las velocidades (gasto por unidad de área transversal) para los mismos segmentos y con la misma periodicidad que la Figura No. 1. Se nota como las corrientes salen del GNS el 3/4/1993 a las 8 a.m. con velocidades máximas cerca del centro del mismo. Sin embargo, las velocidades dos horas más tarde son mayores y positivas (entrando al GNS) en la boca y mínimas en el extremo interior del GNS idealizado. Cuatro horas más tarde, las corrientes son máximas disminuyendo hacia el interior. 
La resaca o marea saliente, posee la misma distribución de corrientes solo que con signo ( 0 dirección) contrario; es decir, las velocidades son mayores a la entrada del golfo y disminuyen hacia el interior. Esto muy probablemente se debe al efecto inercial de las masas de agua que se discute en Murillo (1993) ya la contracción que existe a la entrada del golfo que hace que las masas de agua se aceleren por efectos de la ecuación de continuidad (3)

Se nota como la oscilación se repite cada 12.4 horas, pues está dominada por la componente armónica (Murillo, 1982). Luego de 26.7 horas de cálculos en tiempo real, se han disminuido los modos no permanentes, no armónicos y se observan los efectos de las mareas mayormente.

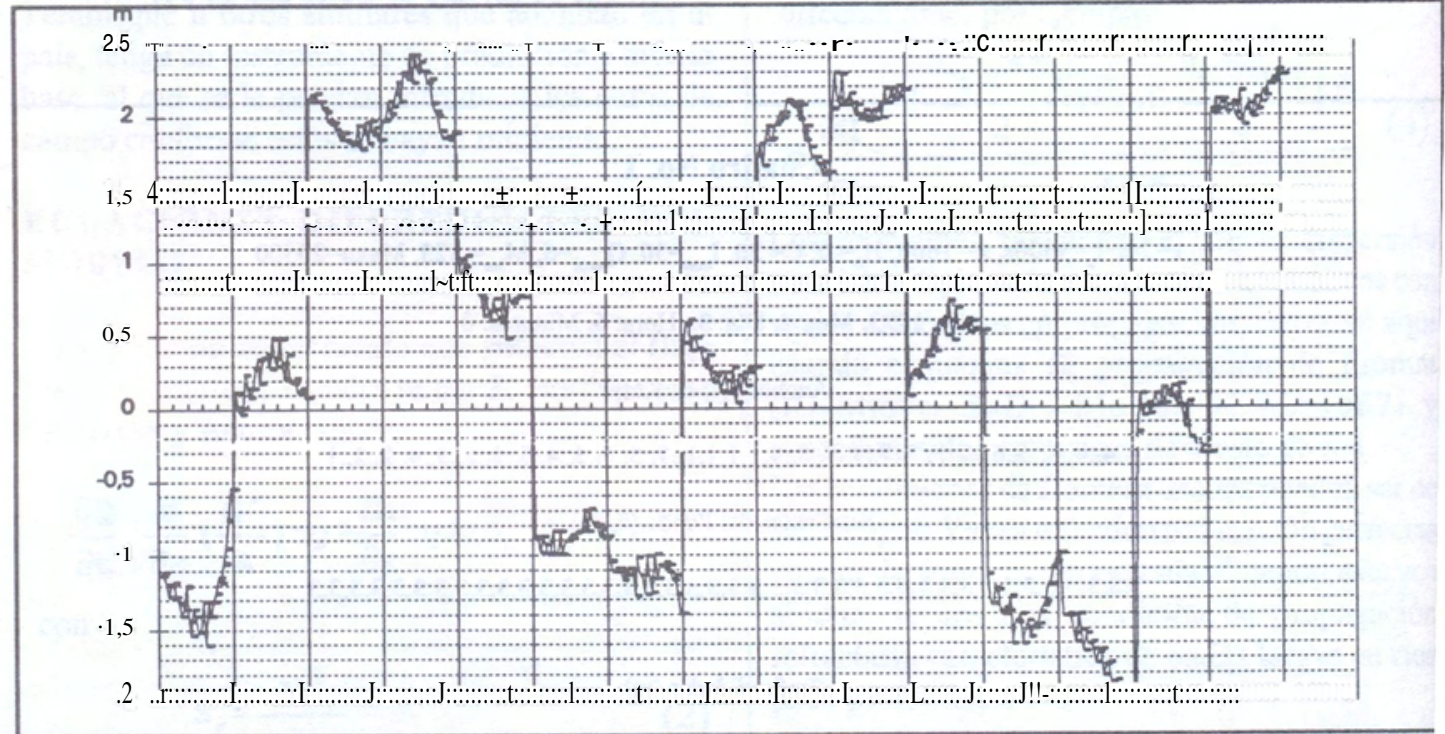

Fig.No.l: Alturas Mareales del Golfo de Nicoya. Cada 123 minutos a partir del período 13, luego del 3/4/1993 a las 6:00 am

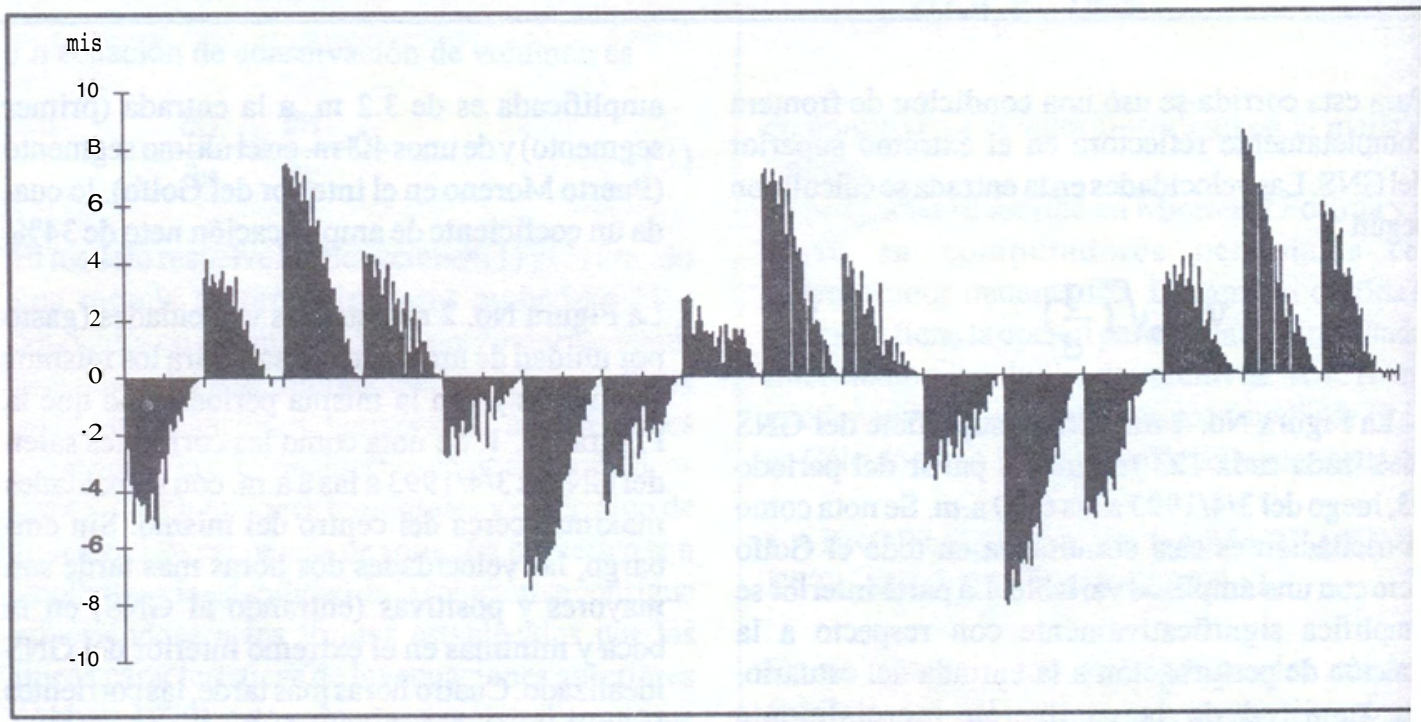

Fig.No.2: Gastos unitarios en el GNS cada 123 min a partir del período 13 luego del del 3/4/1993 a las 6:00 am 


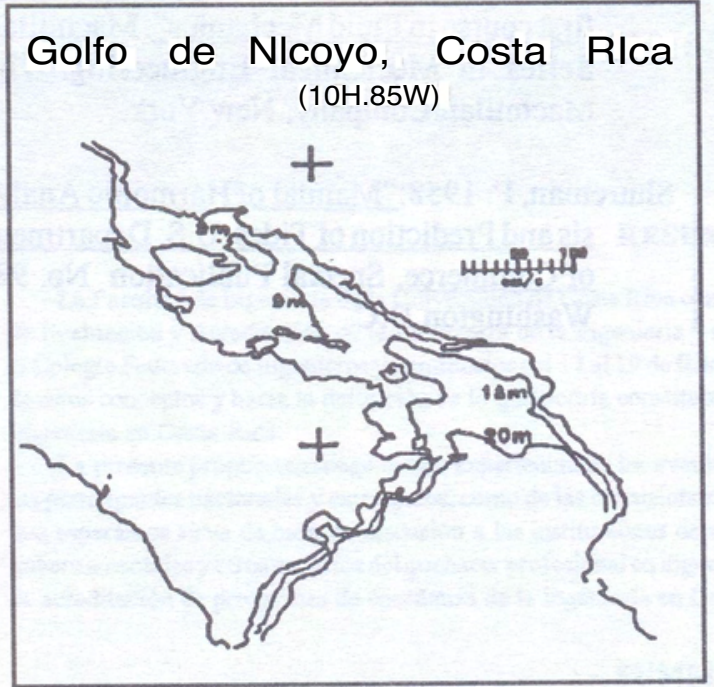

Fig.No.3: El Golfo de Nicoya, Costa Rica

\section{CONCLUSIONES FINALES}

Se introduce un modelo numérico sencillo capaz de reproducir el flujo unidimensional no estable en ríos, canales y estuarios no homogéneos. Se usan diferencias finitas para digitizar las ecuaciones diferenciales y reducirlas a ecuaciones algebraicas que se resuelven mediante métodos implícitos. Se utilizan métodos estándares de la literatura ingenieril para evitar dispersión numérica y asegurar la convergencia y representatividad de los cálculos. El modelo reproduce las características esenciales del flujo conocido para el Golfo de Nicoya en su parte superior (Figura No. 3). Su amplificación y campo de velocidades concuerda con estudios anteriores en sus rasgos generales.

El modelo desarrollado se puede aplicar a ríos y canales pequeflos si se puede obtener información de campo sobre la marea a la entrada, áreas transversales, profundidades, pendientes de fondo $\mathrm{y}$ los coeficientes de Chezy efectivos.

Se considera que el modelo puede ser de gran utilidad para estudiar la problemática relacionada con el desarrollo y utilización de los recursos naturales del río Tempisque en la provincia de Guanacaste, Costa Rica.

\subsection{Simbología}

A: Area transversal de una sección del río.

b: Ancho

C: $\quad$ Coeficiente de fricción de Chezy

$\mathrm{d}$ : Profundidad media

\: Factor de declinación nodal para la componente armónica i

g: Aceleración de la gravedad

$\mathrm{H}: \quad$ Altura de la marea

$\sim$ : $\quad$ Altura de la marea a la desembocadura

del río.

Q : $\quad$ Gasto

$\mathrm{R}_{\mathrm{h}}$ : $\quad$ Radio Hidráulico

$\mathrm{S}_{\mathrm{f}}$ : Gradiente de la línea de energia hidráulica.

So : $\quad$ Gradiente de la cama del río.

T: $\quad$ Período de la marea (44,Z80 segundos)

$\mathrm{V}: \quad$ Velocidad de la corriente

$\mathrm{V}_{\mathrm{mu}}$. Velocidad máxima de la corriente

$\left(\mathrm{V}_{\mathrm{o}}+\mathrm{u}\right)$ : Argumento de equilibrio en Greenwich.

i : Fase local del componente.

\section{BmLIOGRAFÍA}

Abbott, M.B., 1979: "Computational Hydraulics. Elements of the theory of Free Surface Elows". International Institute for Hydraulic and Environmental Engineering, Delft and theDanishHydraulic Institute, Horsholm. Pitman Publishing Lirnited. London, 324 pp.

Murillo, L.M., 1982:"Modelling tidal circulation and dispersion in the Gulf ofNicova. Costa Rica". Oregon State University. MSc. Thesis. Department ofCivil Engineering and School of Oceanography. $186 \mathrm{pl}$.

Murillo, L.M., 1991:"La circulación de las Mareas en el Golfo de Nicoya". Tecnología en Marcha. Vol. 10 (4). p. 51-76. 
Murillo, L.M., 1993 :"La predicción contínU3 de las mareas para las Costas Costarricenses para los aftos 1991-2000". Tecnología en Marcha. Vol. 11 (4). p. 45-48.

Richtmyer R,D., Morton, K.W., 1967: "Difference Methods for Initial- Value Problems". Wiley-Interscience, $405 \mathrm{pp}$.
Sabersky RH., Acosta A.J., 1964:"Fluid Flow. A first course in Fluid Mechanics". Macmillan Series in Mechanical Engineering. The Macmillan Company, New York.

Shureman, P. 1958:"Manual ofHannonic Analy. sis and Prediction ofTides. US Department of Commerce. Special Publication No. 98, WashingtonD.C. 